\title{
Passer des procédures aux processus : l'appropriation du management 0.4 par la qualité dans un hôpital
}

\author{
Régis Martineau \\ Enseignant-chercheur \\ Groupe ESC Troyes, \\ 217 Avenue Pierre Brossolette, 10000 Troyes
}

\section{Résumé}

Les procédures de certification et d'accréditation des établissements de santé français ont débuté il y a maintenant plus de dix ans. Sans constituer de réels échecs, elles rencontrent toutefois quelques difficultés, certaines pratiques et certains outils ne parvenant pas à s'imposer. Cet article présente les résultats d'une recherche, menée au sein d'un Centre Hospitalier Régional Universitaire, visant à identifier les raisons de ces difficultés. II apparaît que dans cet établissement ce ne sont pas des problèmes d'adaptation ou d'adhésion à la démarche qui soient les plus prégnants, mais plutôt la persistance de schèmes familiers faisant obstacle aux approches transversales et processuelles portées par le management par la qualité.

(c) 2012 IDMP/Lavoisier SAS. Tous droits réservés

Mots clés : Appropriation, Procédure de certification, Qualité, Hôpital, Schème.

\section{Abstract}

From procedures to processes: quality management appropriation within a hospital. Quality certification and accreditation has been a challenge for French healthcare organizations for over a decade. Without constituting real failures, they meet however some difficulties, certain practices or tools having difficulties in being established. This article presents the results of a research, conducted within a regional university hospital, to identify the reasons for these difficulties. It seems that it is not staff adaptation or adhesion to the approach that are the most problematic, but rather the obstinacy of familiar schemas putting obstacles to the transverse approaches demanded by quality management.

(c) 2012 IDMP/Lavoisier SAS. Tous droits réservés

Keywords: Appropriation, Quality management, Health care, Schema.

*Auteur correspondant : regis.martineau@get-mail.fr

doi:10.3166/pmp.29.215-232 @ 2012 IDMP/Lavoisier SAS. Tous droits réservés 


\section{Introduction}

Depuis plus de vingt ans maintenant, l'environnement des hôpitaux français connaît des mutations économiques, technologiques, socioculturelles et géopolitiques. En réponse, de nombreuses réformes ont été menées au sein des organisations hospitalières (mise en place du budget global, puis de la tarification à l'activité, la « T2A »; contractualisation avec les Agences Régionales d'Hospitalisation; départementalisation ; mise en place des pôles avec les plans Hôpital 2007 et Hôpital 2012 ; etc). Ces différents mouvements ont favorisé l'émergence d'un hôpital «stratège» (Contandriopoulos et Souteyrand, 1996; Valette, 1996), soumis à un environnement désormais concurrentiel. Pour encadrer ces réformes, l'État crée un quasi-marché en se comportant comme un acheteur de l'offre des établissements de soin (Halgand, 2003). Les dispositifs mis en œuvre pour encadrer les établissements de santé peuvent être analysés comme une réponse aux problèmes posés par les phénomènes d'asymétrie d'information entre l'État et les hôpitaux (Cueille et Renucci, 2000). Parmi ces dispositifs, l'introduction d'un management par la qualité est un élément central, qui mobilise beaucoup de temps, de moyens et d'énergie.

L'État s'appuie donc sur la procédure de certification pour encadrer et contrôler la qualité de la prise en charge fournie par les établissements de santé. Procédure obligatoire pour tous les établissements de santé français, qu'ils soient privés ou publics, elle s'étale sur des cycles de cinq années, et vise à vérifier la conformité des établissements de santé à certaines normes de qualité, établies et décrites dans un manuel de certification et d'accréditation. Débutée au début des années 2000, actuellement s'achève donc la troisième évaluation. Elle « a pour objectif de s'assurer que les établissements de santé développent une démarche d'amélioration continue de la qualité et de la sécurité des soins délivrés aux patients. » (Manuel V2). Près de dix ans après, il apparaît important de s'interroger sur l'appropriation de cette démarche par les établissements de santé, et plus précisément par les acteurs censés la mettre en œuvre, les professionnels de santé.

Sans constituer de véritables échecs, des travaux portant sur les procédures de certification dans les hôpitaux évoquent l'existence de phénomènes de résistance et de couplage ritualisé (Lozeau, 2004), de cassure post-accréditation (Weill, 2001), ou encore d'un manque d'attribution de sens par les personnels aux outils (Fraisse et al., 2003). La version 2 de la démarche de certification a eu lieu, et la version suivante dite «V2010»s'achève actuellement, mais on en sait pourtant assez peu sur les résistances et les difficultés qu'elle a pu occasionner et son appropriation par les professionnels de santé. C'est pourquoi l'étude de cas exposée ici vise à apporter des éléments de réponse en rapportant l'expérience d'un hôpital ayant satisfait à la deuxième procédure de certification. La procédure de certification (dans sa deuxième version) a-t-elle permis l'introduction de pratiques de management par la qualité dans l'hôpital étudié ?

Tout d'abord, nous qualifions la procédure de certification de manière théorique en ayant recours à la notion de dispositif de gestion. Nous montrons comment ce dispositif décline les principes du Total Quality Management (TQM.). Ensuite, les principaux constats issus de l'étude sont présentés puis discutés. 


\section{La procédure de certification comme dispositif de gestion}

Pour appréhender l'appropriation de la procédure de certification, il faut se placer du point de vue des professionnels de santé censés se l'approprier. Or la manière dont se présente la procédure concrètement aux personnels de santé est très complexe. Si le manuel est clair, il est en revanche difficile de décrire les manifestations concrètes de la procédure de certification, tant elle constitue un ensemble complexe, hétéroclite, de textes et de normes à respecter (le manuel de certification), qui appelle la mise en place d'outils (procédures, systèmes informatiques, outils de gestion des risques, etc.) de nouvelles pratiques (la confidentialité, la traçabilité, l'hygiène, l'évaluation des pratiques professionnelles, etc.), et de démarches plus ponctuelles (réunions, groupes de travail, autoévaluation, visite des experts etc.). C'est pourquoi le recours à la notion de dispositif de gestion va permettre d'éclaircir cet aspect. La procédure constitue un dispositif de gestion au sens de De Vaujany (2005), c'est-à-dire un ensemble d'éléments de design organisationnel porté par une intention stratégique, et qui vise à intégrer les outils de gestion de façon cohérente et dans le respect de certaines règles de gestion. Le dispositif de gestion n'est pas un outil de gestion, c'est un ensemble plus complexe porteur de sens qui véhicule des artefacts, des méthodes, des règles et des pratiques.

Ce travail s'inscrit donc dans la lignée de recherches portant sur les instrumentations de gestion et qui considèrent que, loin d'être des objets simples, les instruments de gestion s'inscrivent au contraire pleinement dans les problématiques organisationnelles, et entrent en interaction avec l'action collective (Berry, 1983; Hatchuel et Weil, 1992; Moisdon, 1997 ; David, 1998; Gilbert, 1998; Lorino, 2002 ; De Vaujany, 2005 ; Grimand, 2006 ; Lorino et al., 2011). Ces recherches ont permis de réfléchir les outils, en évitant une vision réductrice qui considérerait les outils comme de simples vecteurs de rationalisation des pratiques, prolongements de la volonté claire du décideur, ne posant pas de problèmes spécifiques, en quelques sortes «désincarnés». Au contraire, les outils posent des questions d'ordre éthiques, politiques, sociales, et ergonomiques. La rencontre entre une organisation est, dans ce courant de recherche, complexe et imprévisible.

Il faut donc voir les outils non comme de simples choses, mais bien plutôt comme des grilles de lecture de l'organisation, s'apparentant à un langage, une manière de parler l'organisation :

"L'outil de gestion se veut essentiellement lecture de l'entreprise en termes économiques (au sens large du terme économique : évaluatif d'une valeur créée comme réponse à un système d'objectifs et de contraintes). L'outil de gestion se présente comme traduction du système d'activités polymorphes de l'entreprise dans un langage d'évaluation par rapport à un jeu de finalités, un système de valeurs à satisfaire.» (Lorino, 2002 : 20).

Le management par la qualité va ainsi traduire la complexité organisationnelle hospitalière dans un langage normé d'évaluation de la qualité, se présentant aux acteurs sous la forme de divers outils plus ou moins formalisés.

Pour appréhender le processus d'appropriation complexe qui va mener à l'usage d'un dispositif de gestion, De Vaujany (2005) propose trois perspectives à prendre en compte : une perspective rationnelle, qui représente le point de vue de la régulation de contrôle (concepteurs, et diffuseurs); la perspective sociopolitique, du point de vue des relations 
sociales (utilisateurs finaux et processus sociologiques); et la perspective psychocognitive, du point de vue des savoirs et des apprentissages (utilisateurs finaux qui apprennent). La première perspective relève de la régulation de contrôle, la deuxième et la troisième de la régulation autonome, en référence aux travaux de Reynaud (1988). Selon la perspective retenue, l'outil ou l'objet de gestion acquiert une nature différente.

Dans une perspective rationnelle, l'outil est un vecteur de rationalisation de l'organisation et du travail; dans une perspective sociopolitique l'outil peut servir de valorisation, de rhétorique ou d'influence; enfin, dans une perspective psycho-cognitive, l'outil est un support d'apprentissage, d'affect ou de traitement de l'information. Il faut actionner les trois regards sur ce phénomène complexe (rationnel, sociopolitique et psychocognitif). Il faut d'abord appréhender l'appropriation du point de vue des concepteurs-formateurs comme un processus à optimiser, à corriger (perspective rationnelle); puis « adopter le point de vue des utilisateurs afin de comprendre comment les objets peuvent gêner ou servir leurs intérêts en fonction de leurs mises en actes » (De Vaujany, 2005 :29) (perspective sociopolitique); enfin comprendre l'appropriation comme un apprentissage parfois difficile par lequel l'individu passe afin de rendre un objet de gestion propre à un usage. Par exemple, Dechamp et Romeyer (2005) ont appliqué cette grille de lecture à propos de l'appropriation du concept de gestion par les médecins et ont montré que l'appropriation s'est faite par les discours corporatistes (syndicats, etc.) plutôt que par le discours officiel. Cela signifie que la perspective sociopolitique de l'appropriation a fortement opéré, dans le sens où le dispositif d'appropriation rationnelle initialement prévu par la réforme a été détourné par les intérêts corporatistes d'une communauté.

De Vaujany notait un certain «ide dans la littérature managériale (De Vaujany, 2005 :231) en ce qui concerne l'étude des outils de gestion dans une perspective sociopolitique et psychocognitive. De même, Grimand (2006) constate la domination de la perspective normative et rationnelle de l'appropriation qui serait censée aller de soi, en vertu du principe selon lequel « l'intendance suivra » (Grimand, 2006 :14). L'étude qui suit vise donc à appréhender les trois perspectives, en portant une attention particulière aux représentations et comportements des professionnels de santé.

\section{Méthodologie d'observation de la procédure de certification}

Après avoir présenté l'étude de cas réalisée au sein d'un CHRU, nous présenterons les principaux résultats de la recherche avant de les discuter.

\subsection{La procédure de certification, une déclinaison des principes du Total Quality Management}

Du point de vue de la régulation de contrôle (la Haute Autorité de Santé, l'HAS), la procédure de certification traduit dans le domaine de la santé les principes de base du management par la qualité. Le TQM. a pour objectif la satisfaction de l'ensemble des parties prenantes de l'entreprise : clients, actionnaires, salariés, fournisseurs et collectivités. La norme ISO 9000 (2005) définit huit principes du management par la qualité : orientation client, leadership, implication du personnel, approche processus, management par approche système, amélioration continue, approche factuelle pour la 
prise de décision, et relations mutuellement bénéfiques avec les fournisseurs. Ces principes se retrouvent pour la plupart adaptés au vocabulaire de l'hôpital dans le manuel V2 de l'HAS (tableau 1), avec une focalisation particulière sur les principes d'orientation client (on parle alors du patient), sur le leadership (l'importance de l'implication de la direction de l'hôpital), sur l'implication des professionnels de santé, sur l'amélioration continue (démarche évolutive et pérenne), et sur l'approche processus (avec la notion de parcours du patient).

Tableau 1 : Principes du Manuel V2

\begin{tabular}{|l|l|}
\hline $\begin{array}{l}\text { Place centrale } \\
\text { du patient }\end{array}$ & $\begin{array}{l}\text { Il s'agit d'apprécier «la capacité de l'établissement à s'organiser } \\
\text { en fonction des besoins et des attentes du patient » (Manuel V2). } \\
\text { L'ensemble des références et des étapes de la procédure est centré } \\
\text { sur l'appréciation de la prise en charge du patient tout au long de } \\
\text { son parcours dans l'établissement et en lien avec les prestations } \\
\text { complémentaires offertes dans son territoire de santé. }\end{array}$ \\
\hline $\begin{array}{l}\text { L'implication } \\
\text { des professionnels }\end{array}$ & $\begin{array}{l}\text { Tous les professionnels doivent participer activement à la mise } \\
\text { en place de la qualité. Cela passe par la responsabilisation de } \\
\text { chacun. }\end{array}$ \\
\hline $\begin{array}{l}\text { Amélioration } \\
\text { du service médical rendu } \\
\text { au patient }\end{array}$ & $\begin{array}{l}\text { Il s'agit d'une démarche pragmatique qui procède par } \\
\text { améliorations successives objectivées par des mesures, visant à } \\
\text { rechercher continuellement l'efficacité et l'efficience. }\end{array}$ \\
\hline La sécurité & $\begin{array}{l}\text { C'est une des attentes principales des patients. Il s'agit ici de } \\
\text { respecter les réglementations et de mettre en place un système } \\
\text { permettant d'identifier et de prévenir les risques. }\end{array}$ \\
\hline Une démarche pérenne & $\begin{array}{l}\text { La certification, par son caractère itératif, vise l'amélioration } \\
\text { continue. L'HAS veille à la mise en place de démarches pérennes. }\end{array}$ \\
\hline Une démarche évolutive & $\begin{array}{l}\text { Les objectifs ne sont pas fixés une fois pour toutes et évoluent } \\
\text { en fonction des résultats, de l'environnement et des attentes } \\
\text { des patients. }\end{array}$ \\
\hline
\end{tabular}

La procédure demande une auto-évaluation par les acteurs de l'hôpital eux-mêmes, avant que des experts visiteurs de l'HAS ne viennent sur place pour vérifier que les exigences du manuel sont respectées. Ces visites donnent lieu à des recommandations et/ou des réserves, et peuvent rendre nécessaire une contre visite sur des points précis. Elle s'appuie sur un manuel de certification et d'accréditation évolutif : à une première version dite «V1»a succédé une deuxième, dite «V2», puis une troisième, dite «V2010».

Ce manuel est divisé en deux parties, la première rappelant les fondements et principes de l'accréditation; et la deuxième développant en quatre chapitres les dispositifs à mettre en œuvre et les principaux points évalués par les auditeurs. Chacune des références est précisée avec l'objectif qui lui est assigné, les éléments d'appréciations pris en compte par les auditeurs, les documents et les personnes ressources. On trouvera en annexe une description précise du manuel V2. 
En analysant les documents fournis par l'HAS, on peut reconnaître six fonctions sous jacentes censées être appropriées par les professionnels de santé :

- Améliorer : la procédure de certification a pour objectif d'améliorer la qualité et la sécurité des soins délivrés aux patients.

- Normaliser : en s'appuyant sur des mesures objectives en termes d'efficacité et d'efficience, l'HAS contrôle et évalue les résultats obtenus par les établissements de santé. Elle vise donc à faire appliquer des bonnes pratiques.

- Coordonner et communiquer : l'HAS entend mobiliser et faire participer aussi bien les professionnels que les usagers et la Direction de chaque établissement, en les faisant travailler ensemble, et en faisant mieux circuler l'information

- Faire réfléchir : par le biais de l'autoévaluation notamment, elle vise à amener les praticiens à réfléchir sur leurs pratiques.

- Réguler : la procédure vise à décider des autorisations en matière d'allocation de moyens et de ressources aux hôpitaux.

- Assurer la qualité vis-à-vis de l'extérieur : la procédure de certification vise à assurer la qualité de la prise en charge du patient vis-à-vis du grand public, elle a pour but de lui assurer un certain niveau de qualité et de sécurité des soins (suivant les principes de l'assurance qualité).

Ces éléments présentent la procédure telle qu'elle devrait être appropriée par l'ensemble des professionnels de santé (perspective rationnelle de l'appropriation). Le cadre méthodologique retenu vise donc à estimer dans quelle mesure la procédure de certification réellement mise en œuvre s'écarte ou se rapproche de ces objectifs fixés par l'HAS

\subsection{Présentation du cas et méthodologie}

Pour cela, cette recherche s'appuie sur une étude de cas (Yin, 2003), l'avantage de cette méthode étant d'approfondir considérablement la connaissance d'un terrain, ce qui est particulièrement indiqué pour l'étude de l'appropriation d'un dispositif de gestion. L'hôpital étudié est un établissement public de santé multisite et constitue une très grande organisation, rémunérant plus de 6000 personnes, effectuant plus de 100 métiers différents, et d'une capacité d'environ 2000 lits et places.

Une première étude exploratoire a été menée, combinant présence sur le terrain, phases d'observations, présence lors de réunions (présentations de la direction, groupes de travail) et entretiens semi-directifs ; à laquelle a succédé une seconde phase terrain principalement consacrée à des entretiens semi-directifs. L'ensemble de l'étude terrain, effectuée dans le cadre d'un travail doctoral, a été menée entre mars 2005 et mars 2009, a permis le recueil de 50 entretiens enregistrés et retranscrits d'une durée moyenne d'environ une heure (42 pour la seconde phase terrain, voir tableau 2), complétés par le recueil de nombreuses observations et données primaires. L'ensemble du matériel recueilli concerne donc la période qui a suivi la deuxième procédure d'accréditation, l'hôpital ayant été audité début 2006, ce qui explique pourquoi l'analyse porte sur le manuel V2, en vigueur durant la période d'observation.

Les données secondaires externes sont constituées principalement des documents fournis par l'HAS (manuels de certification, comptes rendus, manuels méthodologiques à l'usage des personnels) qui ont permis de décrire précisément la procédure 
de certification telle que «prescrite» par l'HAS, et ensuite de les comparer avec les représentations des acteurs. Les données secondaires internes sont constituées par des observations et des comptes rendus de réunions et de groupes de travail, mais aussi par des traces laissées par l'usage d'outils de gestion (fiches de signalement d'incident, cartographies de processus, etc.). Les professionnels de santé rencontrés ont été principalement des cadres de santé, des médecins et des paramédicaux. En effet, le choix a été fait de focaliser sur le travail quotidien des acteurs de santé, puisque la qualité vise l'implication de tous les personnels. Ainsi, l'étude des implications concrètes et quotidiennes de la procédure de certification au plus près du terrain a été privilégiée au détriment de l'analyse du sommet stratégique (même si le dialogue avec la Direction a été constant). Le tableau 2 présente quelques caractéristiques de l'échantillon des répondants et permet de voir qu'une diversité dans le profil des répondants a été recherchée, allant de personnes ayant eu des formations et des responsabilités dans le domaine de la qualité, alors que d'autres pas du tout.

Tableau 2 : Caractéristiques des répondants

\begin{tabular}{|l|l|c|}
\hline Caractéristique & Attribut & $\begin{array}{c}\text { Nombre } \\
\text { de répondants }\end{array}$ \\
\hline \multirow{4}{*}{$\begin{array}{l}\text { Catégorie } \\
\text { professionnelle }\end{array}$} & Administratif (cadres de santé principalement) & 20 \\
\cline { 2 - 3 } & $\begin{array}{l}\text { Paramédicaux } \\
\text { (infirmières, manipulateurs radio, aides soignants, etc.) }\end{array}$ & 16 \\
\cline { 2 - 3 } & Médecins & 6 \\
\hline \multirow{4}{*}{ Ancienneté } & Entre 0 et 3 ans & 6 \\
\cline { 2 - 3 } & Entre 3 et 10 ans & 7 \\
\cline { 2 - 3 } & Plus de dix ans & 29 \\
\hline \multirow{4}{*}{$\begin{array}{l}\text { Expérience en } \\
\text { qualité }\end{array}$} & Forte & 12 \\
\cline { 2 - 3 } & Moyenne & 13 \\
\cline { 2 - 3 } & Aucune & 23 \\
\hline \multirow{3}{*}{$\begin{array}{l}\text { Formation } \\
\text { en qualité }\end{array}$} & Forte & 8 \\
\cline { 2 - 3 } & Moyenne & 11 \\
\cline { 2 - 3 } & Aucune & 13 \\
\hline
\end{tabular}

Les entretiens ont été menés de manière individuelle, en assurant aux répondants une confidentialité totale, en soulignant l'indépendance du chercheur, et en proposant un droit de révision sur les propos tenus, afin d'instaurer un «climat de confiance relatif» (Grawitz, 1996). Les entretiens visaient à répondre à quatre questions principales (tableau 3 ). 
Tableau 3 : Guide d'entretien

\begin{tabular}{|l|l|}
\hline Question clé & Questions types posées en entretien semi-directifs \\
\hline $\begin{array}{l}\text { Les usages sont-ils } \\
\text { effectifs? }\end{array}$ & $\begin{array}{l}\text { Quels sont les impacts concrets de la procédure de certification? } \\
\text { Quel impact sur vos habitudes de travail? } \\
\text { Croisement avec des données empiriques observées } \\
\text { (réunions, documentation). }\end{array}$ \\
\hline $\begin{array}{l}\text { Quelles sont les } \\
\text { caractéristiques perçues } \\
\text { du dispositif par les } \\
\text { utilisateurs? }\end{array}$ & $\begin{array}{l}\text { La procédure est-elle pratique ? Est-elle bien faite? } \\
\text { Avez-vous des critiques à lui faire? } \\
\text { Auriez-vous des suggestions pour l'améliorer? }\end{array}$ \\
\hline $\begin{array}{l}\text { Les utilisateurs } \\
\text { adhèrent-ils au principe } \\
\text { du dispositif ? }\end{array}$ & $\begin{array}{l}\text { La procédure est-elle utile? } \\
\text { Pensez-vous qu'elle apporte une amélioration? } \\
\text { Trouvez-vous que cette procédure a sa place au sein } \\
\text { de cette institution? }\end{array}$ \\
\hline $\begin{array}{l}\text { Quelles sont les } \\
\text { fonctions attribuées } \\
\text { au dispositif par } \\
\text { les utilisateurs ? }\end{array}$ & $\begin{array}{l}\text { À quoi sert concrètement la procédure? } \\
\text { A-t-elle réglé certains dysfonctionnements? }\end{array}$ \\
\hline
\end{tabular}

Enfin, une analyse de contenu a été menée sur les entretiens effectués, qui ont été retranscrits puis codés à l'aide du logiciel Nvivo 8.0. Ce logiciel est dédié à l'analyse de données qualitatives assistée par ordinateur : il se rapproche de l'analyse qualitative papiercrayon, mais apporte davantage de rapidité et de flexibilité dans le traitement des données. Cela permet de coder les verbatim autour d'unités de sens, qui se constituent en un arbre hiérarchique. Les données collectées ont donc fait l'objet, à l'aide ce logiciel, d'une analyse de contenu à partir des thèmes prédéfinis (Miles et Huberman, 1991).

Les données ont été découpées selon deux axes. Le premier a concerné les éléments contextuels portant sur le CHRU en général, puis sur l'individu interrogé en particulier. Le deuxième a concerné la perception de la procédure par les professionnels de santé : des catégories reprenant les quatre questions clés ont été codées (usage effectif; adhésion au principe; caractéristiques perçues du dispositif; fonctions attribuées). Malgré un recoupage des déclarations des répondants avec des données d'observation et de documentation, les résultats exposés ici se basent donc principalement sur du déclaratif. Ce mode de recueil peut paraître limité au regard de l'objet d'analyse qui porte aussi sur des usages concrets. Cependant, le fait de recourir à du déclaratif permet d'accéder aux représentations des acteurs, et s'avère donc un mode de collecte des données partiel, limité, mais en même temps nécessaire.

\section{L'appropriation de la procédure de certification : un problème d'apprentissage?}

Nous avons pu observer l'appropriation de la procédure de certification dans ses différentes perspectives. Même si des difficultés d'appropriation apparaissent, c'est principalement sur l'aspect psychocognitif qu'elles se font les plus prégnantes. 


\subsection{L'appropriation de la procédure de certification dans ses aspects rationnels}

L'analyse des fonctions attribuées par les répondants à la procédure va permettre de comparer le point de vue de la régulation de contrôle (l'HAS) à celui de la régulation autonome (les professionnels de santé) (tableau 4).

Tableau 4 : Fonctions prescrites et fonctions attribuées par les répondants

\begin{tabular}{|c|c|}
\hline $\begin{array}{l}\text { Fonctions prescrites } \\
\text { par l'HAS }\end{array}$ & Les fonctions sont-elles attribuées par les répondants ? \\
\hline Améliorer & $\begin{array}{l}\text { C'est la principale fonction mise en avant par les répondants } \\
\text { pour expliquer leur mise en usage. La procédure de certification } \\
\text { est effectivement vue comme un moyen permettant d'améliorer } \\
\text { concrètement la qualité des soins, et aussi d'optimiser la sécurité des } \\
\text { soins, qui sont les deux objectifs principaux cités par le manuel de } \\
\text { certification. }\end{array}$ \\
\hline Faire réfléchir & $\begin{array}{l}\text { Les acteurs s'interrogent en effet sur les pratiques habituelles de travail } \\
\text { et d'organisation. La procédure permet de prendre du recul, de voir } \\
\text { l'organisation autrement, et de se remettre en cause (notamment via les } \\
\text { groupes de travail). }\end{array}$ \\
\hline Normaliser & $\begin{array}{l}\text { La procédure est en effet vue comme un moyen de se mettre à jour sur } \\
\text { les législations en vigueur. Ensuite, les outils sont mobilisés en tant } \\
\text { qu'outil de formation pour les nouveaux arrivants, notamment dans le } \\
\text { cas des procédures : la formalisation de pratiques de travail admises et } \\
\text { réglementaires permet de mieux expliquer et former les professionnels } \\
\text { de santé. Cette fonction est particulièrement utile dans des services qui } \\
\text { souffrent d'un fort turnover. }\end{array}$ \\
\hline $\begin{array}{l}\text { Coordonner et } \\
\text { communiquer }\end{array}$ & $\begin{array}{l}\text { La procédure permet effectivement de faire participer et d'impliquer } \\
\text { l'ensemble des personnels dans des projets transversaux. Cela remet } \\
\text { en cause les habituels cloisonnements entre services et entre corps de } \\
\text { métiers. Cela permet par ailleurs aux cadres de mobiliser les équipes en } \\
\text { les impliquant davantage. }\end{array}$ \\
\hline Assurer la qualité & $\begin{array}{l}\text { Aucun des répondants ne mobilise explicitement cette fonction comme } \\
\text { argument pour la mise en usage des outils de gestion par la qualité. S'ils } \\
\text { se soucient de l'avenir de l'hôpital et de son image, ce n'est en tous les } \\
\text { cas pas une fonction qu'ils mettent en avant pour justifier de la mise en } \\
\text { usage des outils portés par la certification. }\end{array}$ \\
\hline Réguler & $\begin{array}{l}\text { Le fait que la qualité puisse avoir un lien avec les autorisations en } \\
\text { matière d'allocation de moyens et de ressources n'est pas un aspect mis } \\
\text { en avant par les répondants pour justifier des usages. }\end{array}$ \\
\hline
\end{tabular}

Dans une très large mesure, les répondants adhèrent aux principes portés par la procédure de certification, c'est-à-dire qu'ils lui trouvent du sens, un intérêt, et la jugent cohérente et utile. Les personnels comprennent la nécessité d'une évaluation par une entité externe, et la nécessité d'afficher un niveau de qualité vis-à-vis de l'extérieur. Par ailleurs, ils recon- 
naissent la nécessité de mettre en place des procédures normalisées. Il semble donc que la démarche, bien qu'obligatoire et imposée, soit reçue positivement dans ses principes. Ce constat confirme les résultats d'une précédente étude (Claveranne et al., 2003 : 12), qui constatait que « Globalement, l'accréditation est perçue par l'ensemble des acteurs comme contribuant à l'amélioration de la qualité en général (85\% des personnes rencontrées estiment que l'accréditation contribue à l'amélioration de la qualité) 》.

Bon nombre de répondants apprécient la procédure dans ce qu'elle permet d'améliorer la qualité et la sécurité de prise en charge du patient, de faire réfléchir sur l'organisation, de normaliser et de communiquer entre services qui ne se rencontrent pas habituellement, même si la procédure de certification est souvent ressentie comme relativement complexe, notamment au regard du langage qualité parfois ésotérique, et du travail demandé qui est jugé difficile et coûteux en terme de temps. En revanche, on voit que le côté «assurance de la qualité » vis-à-vis de l'extérieur n'est pas -ou très peu - mentionné par les acteurs : les fonctions de valorisation externe et de régulation ne sont pas invoquées pour justifier des usages. Cela signifie que les professionnels de santé, dans le cours de leurs actions, cherchent à trouver un sens concret aux nouvelles pratiques et outils : à quoi cela sert-il, concrètement, dans la pratique quotidienne ? Sans être en désaccord avec ces objectifs d' «affichage», les personnels n'y voient cependant pas un moteur à son appropriation. Les possibilités offertes par la démarche de réfléchir sur les pratiques de travail et de rencontrer d'autres équipes sont en revanche particulièrement appréciées : "Je trouve que l'autoévaluation, c'est bien, parce que cela permet de prendre le temps de faire un bilan, de se poser, de se dire : "bon voilà où est ce qu'on en est ", et améliorer ce qu'on peut améliorer, et de réfléchir un peu avec les équipes » (un cadre de santé).

\subsection{L'appropriation de la procédure de certification dans ses aspects sociopolitiques}

Dans ses aspects sociopolitiques, l'appropriation mène à des comportements plus ou moins « déviant» et inattendus. La procédure de certification est notamment mobilisée comme moyen de revendication, dans une logique d'acquisition de ressources. Par exemple, certains praticiens profitent de la visite des experts visiteurs pour mettre en avant le manque de moyens, espérant ainsi faire pression sur la direction pour obtenir ces moyens. De manière plus générale, les outils de gestion par la qualité sont souvent l'occasion pour les professionnels de santé de mettre en avant un problème récurrent, un dysfonctionnement, qui est sans doute légitime mais qui dépasse les strictes possibilités d'intervention des qualiticiens et même de l'HAS Par exemple, il n'est pas rare que la nuit, en services d'urgences, une seule infirmière se retrouve à gérer un grand nombre de patients : les outils qualité peuvent alors servir de moyen de pression pour obtenir plus de moyens en personnels, cela peut servir d'argument: "Il y en a, c'est sûr, qui ne se gênaient pas pour le dire devant les experts visiteurs » (un cadre de santé en radiologie). Des comportements «cérémoniels» (qui consistent à feindre un comportement lors de la visite des experts visiteurs) souvent décrits (Lozeau, 2004) existent, comportement qu'illustre assez bien l'anecdote de cette infirmière : «Donc, cette journée-là, on a enlevé le micro-onde et tout ça de la pièce, on a tout emmené dans les soins, parce qu'ils venaient pour l'accréditation... Et, après, dès qu'ils sont passés, on a tout remis dans le réfrigérateur ». 
De même, l'évaluation des pratiques professionnelles (EPP) est parfois mal acceptée par les médecins, qui y voient une perte de pouvoir face au gestionnaire. Ces jeux politiques autour de la procédure de certification ne sont toutefois pas étonnants et ont souvent été décrits dans la littérature. Mais, sans les sous estimer, selon nous, ces difficultés n'apparaissent pas comme la cause profonde des difficultés rencontrées, dans la mesure où certaines nouvelles pratiques s'imposent relativement bien, comme c'est le cas de celles liées à la confidentialité (retrait des noms au lit des patients, retrait des plannings d'occupation des salles, etc.). Pourtant, certaines habitudes paraissaient bien ancrées : "S'il y a 10 ans on m'avait dit qu'il n'y aurait plus de pancarte sur les lits, un truc tout bête, pour la confidentialité, on aurait dit «ce n'est pas possible ». À l'hôpital, on voit tous la pancarte au bout du lit, et çà, çà a disparu partout. » (une cadre de santé aux urgences)

En somme, au-delà de quelques phénomènes de résistance bien connus, la procédure de certification ne se heurte pas à des résistances sociopolitiques insurmontables dans l'hôpital étudié, comme le résume ce médecin : "Les accréditations? Une musique commence à se mettre dans la tête des gens». Toutefois, cette «musique» joue selon nous une partition incomplète.

\subsection{La perspective cognitive de l'appropriation : passer des procédures aux processus}

En effet, ce qui est particulièrement frappant, c'est la difficulté des outils dits « transversaux » à s'imposer. Dans le cas étudié, ces outils, comme l'approche processus, les systèmes de gestion des risques, ou le Dossier Patient Partagé ont beaucoup de mal à s'implanter. Au cours de l'étude, nous avons pu suivre un groupe de travail "processus » chargé de décrire le parcours du patient au niveau global et au niveau local à l'intérieur des services. Mis à part quelques expériences locales, cette description des processus n'a abouti à aucune mise en œuvre concrète (seul un service a été jusqu'à un suivi régulier d'indicateurs, sur huit groupes de travail). Ce médecin va même plus loin : «il y a des processus qui ont été décrits, je ne connais pas de processus qui fonctionne. C'est-à-dire qu'ils ne sont pas utilisés, comme vous dites, comme gestion de l'activité ». Au niveau de l'établissement, la description du parcours patient (pourtant formalisée) n'a pas été utilisée par la direction comme grille d'analyse des dysfonctionnements organisationnels. La deuxième procédure de certification ne semble pas avoir apporté d'évolutions majeures dans le cas étudié. Les personnels de santé (excepté les cadres de santé, qui en perçoivent mieux les enjeux) perçoivent la procédure de certification dans ses aspects les plus « procéduriers » et normalisant.

Ce constat fait écho à un certain nombre de travaux qui ont mis en avant ce problème. En effet, en matière de qualité, il faut distinguer ce qui relève de la « normalisation des pratiques » (aspects réglementaires et procéduriers, visant à assurer que les règles d'hygiène et de sécurité, notamment, sont appliquées), de ce qui relève de l' " approche transversale » (une description de l'activité de l'hôpital en terme de processus, qui décrit le parcours du patient au sein de l'hôpital). Claveranne et al. (2003) ont mis en évidence au cours d'une recherche sur la qualité hospitalière une distinction entre les travaux relevant de la formalisation de pratiques et les actions transversales sur un plan organisationnel. D'autres travaux (Campinos-Dubernet et Jougleux, 2002 ; Boiral, 2002 ; Benezech et Loos-Baroin, 2003; Lozeau, 2004) ont questionné la capacité des démarches qualité à introduire un réel changement organisationnel. Cela renvoie à la différence entre l'Assurance de la Qualité et 
la Qualité Totale : l'assurance de la qualité est fondée sur le principe de surveillance et sur des schémas d'exécution du travail qui obéissent à un principe de conformité à des standards ou à des normes préétablies (Husser, 2006) ; alors que la Qualité Totale préconise l'interaction des processus et des acteurs (Bertezene, 2003; Minvielle, 2003). Derrière le TQM, on trouve donc deux aspects différents : l'un relevant de la formalisation des pratiques; et l'autre, s'appuyant sur une approche transversale de l'organisation. Ce constat est corroboré par d'autres recherches qui ont montré que la diffusion d'une logique de gestion basée sur les processus se heurte à des blocages bien spécifiques aux hôpitaux (Pascal, 2003).

Notre grille de lecture permet de montrer que, selon nous, ce semi-échec est dû non pas à un manque d'adhésion au principe de la démarche, ni à un défaut du dispositif en luimême, mais plutôt à un problème d'apprentissage des schèmes transversaux portés par ces outils. Ce ne sont donc pas tant les aspects rationnels et sociopolitiques de l'appropriation qui posent problème, mais plutôt ses aspects psycho-cognitifs. Les aspects transversaux de la démarche qui rencontrent de réelles difficultés à s'imposer démontrent l'apprentissage difficile d'un nouveau mode de fonctionnement basé sur une nouvelle vision de l'organisation hospitalière et de son fonctionnement. Afin d'approfondir cet aspect psycho-cognitif de l'appropriation, nous avons recours à la notion de «schème» pour éclairer ces difficultés.

\section{Discussion : la nécessité de faire évoluer les schèmes à l’hôpital}

Les résistances aux changements sont souvent vues comme des résistances d'ordre sociopolitiques ou idéologiques, à la manière de celles que produisent les jeux d'acteurs de Crozier et Friedberg (1977), les tactiques locales (De Certeau, 1990), les désaccords de principe face au Logos gestionnaire (Maugeri, 2001 ; Boussard, 2008), etc. Ces cadres théoriques expliquent en partie les comportements cérémoniels ou les abandons de pratiques. Mais si ces phénomènes existent, ils ne résument pas tout. Les difficultés des outils transversaux s'expliquent aussi par une activité hospitalière traditionnellement centrée sur le service, isolée et cloisonnée par rapport au reste de l'organisation. Cette vision est intégrée jusque dans les gestes des professionnels de santé, elle y est littéralement «incorporé». Il ne suffira donc pas de décrire l'hôpital en termes de parcours patient, puis de mettre en place des indicateurs, en exigeant de l' «intendance» qu'elle suive. Car sans la compréhension de la vision transversale qui sous-tend chaque processus et chaque indicateur, les personnels feront semblant sur le court terme et abandonneront la pratique sur le long terme : un outil ne fonctionne que si son utilisateur lui attribue un sens. Les personnels voient l'intérêt de respecter une procédure d'hygiène ou de respecter l'anonymat du patient; ils ont beaucoup plus de mal à voir en revanche l'intérêt de décrire le parcours du patient. Cela est effectivement difficile si l'on n'a pas une vision transversale de l'organisation.

Approfondissons un exemple précis : en matière de gestion des risques, il existe un outil transversal (la fiche de signalement d'incident), disponible partout et utilisable par tous les personnels, qui permet de signaler un événement indésirable, à savoir un incident ou un risque d'incident :

«C'est un outil totalement décloisonnant. Non seulement l'outil en lui-même doit être un outil de décloisonnement, mais en plus nous, on doit l'interpréter et le traiter derrière en terme de processus : c'est pas un service, c'est pas un acteur, 
c'est tout un ensemble d'activités, toute une chaîne d'acteurs qui sont intervenus et qu'il faut comprendre et essayer de voir à quel niveau il faut poser des verrous pour sécuriser mieux notre activité » (un membre de la direction qualité).

Les incidents les plus intéressants pour l'analyse sont les incidents qui révèlent quelque chose sur le fonctionnement de l'hôpital (attente de patients entre deux services, mauvaise livraison des repas ou des médicaments, par exemple). Pourtant, ce sont principalement des chutes de patients, des violences, ou des vols qui sont signalés. La fiche est naturellement plutôt utilisée comme simple élément de signalement (dans une logique de «prendre acte » d'un incident) plutôt que dans une démarche de réflexion globale pour résoudre un problème organisationnel. Pour comprendre l'utilité de cet outil, il faudrait que l'utilisateur «voit» (un minimum) l'organisation comme un tout, et raisonne de manière globale et transversale.

Le recours à la notion de schème permet précisément d'éclairer cet aspect psychocognitif de l'appropriation peu traité dans la littérature en management. Ce concept de schème a connu des évolutions depuis la conceptualisation de Piaget (1967). Chez cet auteur, les schèmes « constituent des moyens du sujet à l'aide desquels il peut assimiler les situations et les objets auxquels il est confronté » (Rabardel, 1995 :79). Le schème est donc un moyen d'assimilation, qui, en sa forme la plus simple, est la tendance de toute conduite à se conserver. Pour Vergnaud (2001), les schèmes sont les formes stables d'organisation de l'activité, face à des situations d'une certaine variété. Les schèmes «familiers » (Rabardel, 1997) des personnels de santé, qui sous-tendent leur manière d'agir et de penser l'organisation hospitalière, sont trop éloignés des schèmes «génériques » portés par les outils transversaux. Par contre, les outils du type formalisation des pratiques ont moins de difficultés à s'imposer car ils correspondent davantage aux schèmes familiers. Le principal problème sera donc un problème d'apprentissage (chez Piaget, l'apprentissage passe précisément par l'accommodation et l'assimilation de nouveaux schèmes).

Plus le schème d'utilisation générique de l'instrument est familier aux utilisateurs, plus l'instrument sera appliqué dans le sens prévu par les concepteurs, ce qui explique pourquoi les aspects «procéduriers» de la démarche fonctionnent relativement bien. On a pu constater que la rédaction de procédures ainsi que la confidentialité sont deux aspects qui sont relativement bien acceptés et mis en œuvre par les professionnels de santé : ces outils sont aujourd'hui effectifs, et relativement bien rentrés dans les habitudes de travail. Ils s'inscrivent dans la continuité, le moment de l'accréditation ne semblant pas influencer l'effectivité de ces outils. Nous pouvons constater aussi que ce sont deux outils dont les modes de fonctionnement sont déjà ancrés dans les habitudes de travail des praticiens : discrétion sur les informations relatives au patient pour la confidentialité; réglementation légale des pratiques pour les procédures.

Pour utiliser ces outils, les acteurs mobilisent un schème «familier» :

- « les protocoles, (...) pour nous c'est de la routine, c'est rentré dans les us et coutumes, c'est une recette de cuisine pour chaque acte » (une infirmière);

- les outils qui mobilisent des schèmes «non familiers » auront plus de mal à être appropriés par les utilisateurs : les approches transversales ont plus de mal à s'imposer, comme le souligne ce médecin, plus familier des démarches qualité :

"On peut créer ce genre de chose, c'est peut être très bien, mais à la base les manipulateurs qui sont dans la production d'actes sont très distants de ce genre de chose. 
Ce n'est pas leur préoccupation. Ils sont assez distants de tout ce qui est description de processus, parce que c'est pas leur souci quotidien, c'est pas leur problème. »

Cela est principalement dû au cloisonnement entre services, si caractéristique de la structure hospitalière :

«Jusqu'à présent on a toujours fonctionné, en tout cas dans les hôpitaux publics, sur ce qui se passe chez nous, intra muros au service. Après, savoir que la radiologie ils ont telle problématique, que les laboratoires vont avoir besoin de tant d'étiquettes, parce que sinon ils vont être gênés et que s'ils sont gênés on perd encore une demi-heure... (...). Et cette vision-là des problématiques des autres services, elle est pas partagée en fait. Nous, on voit bien nos problèmes à nous, mais les problèmes des autres... Et, du coup, ça manque de fluidité dans la prise en charge d'une personne. » (un cadre de santé)

Précisons que les personnels qui ont eu des formations en qualité sont ceux qui sont les plus familiers avec le schème transversal : ils sont ceux qui mettent le doigt sur le problème et le déplorent, prenant parfois des initiatives locales, mais ne parviennent pas à faire vivre des approches processus, principalement du fait de l'incompréhension de leurs collègues.

Ainsi, à l'avenir, l'enjeu principal des procédures de certification sera, à notre sens, de réussir à faire approprier par les personnels de réels outils transversaux, qui, seuls, sont à même d'enclencher de vrais changements dans les structures organisationnelles, et un réel management par la qualité. En d'autres termes, le changement des pratiques induit par les démarches qualité ne va pas jusqu'à remettre en cause les schèmes d'utilisation «habituels », «familiers» des professionnels de santé. Habitués à réfléchir en termes de services et de procédures locales, ils ont des difficultés à mobiliser des outils qui demandent une vision transversale de l'organisation. Si les démarches qualité ont permis de gagner en sécurité dans les actes quotidiens, on peut douter de leur capacité à régler les dysfonctionnements organisationnels des hôpitaux. Une cadre de santé exprimait son enthousiasme suite à la visite d'un autre hôpital, en Belgique, qui a « des parcours de soin qui sont identifiés. (...) Avec un périmètre qui est défini, les acteurs qui sont identifiés, les interfaces qui sont prévues... C'est magique. Vu comment ils nous l'ont décrit, ça a l'air formidable. »

C'est donc selon nous l'aspect transversal du management par la qualité qui fait défaut pour l'instant. Du point de vue méthodologique, il est évidemment vain de tenter de généraliser ce constat à d'autres établissements, ni même à l'ensemble de la très grande et complexe organisation hospitalière étudiée ici. Ce constat n'a de valeur que dans la mesure où il avance une explication peu explorée sous cet angle des difficultés d'appropriation du management par la qualité, davantage envisagées dans les perspectives rationnelles et sociopolitiques.

\section{Conclusion}

La procédure de certification est reconnue comme légitime par les personnels, même si elle donne lieu, de manière presque «normale», à des comportements cérémoniels, des abandons de pratique, quelques mécontentements autour de sa mise en œuvre, et des jeux politiques. Mais le problème ne réside pas selon nous dans la perspective rationnelle et sociopolitique de l'appropriation. C'est plutôt dans ses aspects psychocognitifs qu'il réside, car les outils transversaux ont plus de mal à s'imposer que les outils procéduriers. C'est 
sur cet aspect que la procédure de certification échoue à diffuser ce principe essentiel du management par la qualité, et le recours à la notion de schème permet d'éclairer ce réel problème d'apprentissage. Ce constat permet de mettre en avant l'aspect psychocognitif de l'appropriation, dimension moins explorée habituellement; toutefois il ne surprendra pas le qualiticien, qui sait que c'est cette vision transversale de l'organisation est l'une des principales originalités du management par la qualité, et donc un de ses principaux défis.

La procédure de certification et d'accréditation des établissements améliore la qualité des soins délivrés aux patients dans le sens où elle améliore le respect des bonnes pratiques, en favorisant la formalisation et la réflexion sur les pratiques existantes. En revanche, dans l'hôpital observé, elle ne bouleverse pas encore fondamentalement l'organisation hospitalière : un management plus transversal et basé sur la gestion par les processus reste à mettre en place. Il conviendra que tous les acteurs s'approprient ce schème qui se réfère à une vision transversale de l'organisation, afin qu'ils fassent sens de ces démarches. En effet, sans la coopération de la totalité des professionnels de santé, les habituels dysfonctionnements hospitaliers, qui se produisent souvent à l'interface entre les services, continueront d'avoir lieu. Le plus difficile reste donc à faire pour l'HAS

Pour vaincre cette difficulté et mettre en place un réel management par la qualité, l'HAS pourra, et devra s'appuyer sur les cadres de santé, qui possèdent les clés de la réussite de par leur position de traducteur au sein de l'organisation. Il doit réussir à convaincre de l'intérêt des approches processus et transversales, et donc trouver des moyens de «raconter» la transversalité en utilisant des termes concrets qui font sens pour les professionnels localement, comme le résume ce cadre de santé : "Il faut traduire les termes qualité en termes opérationnels, et je pense que la qualité en général aurait tout intérêt à ne pas utiliser les termes de qualité, en fait. Pour que les gens aient l'impression qu'on leur parle de ce qu'ils font. »

\section{Bibliographie}

BENEZECH D. et LOOS-BAROIN J., (2003). Le processus de certification ISO 9000 comme outil d'apprentissage organisationnel, Revue Sciences de gestion 36, 11-41.

BERRY M., (1983). Une technologie invisible : l'impact des instruments de gestion sur l'évolution des systèmes humains, Centre de recherche en gestion de l'école Polytechnique.

BERTEZENE S., (2003). Les enjeux stratégiques de la qualité dans les établissements médico-sociaux : le cas des établissements d'accueil pour personnes âgées, XII Conférence de l'AIMS, les Côtes de Carthage, 3,4, 5 et 6 juin 2003 .

BOIRAL O., (2002). ISO 9000 : de l'impératif commercial au rite de passage organisationnel, $X I^{\mathrm{e}}$ Conférence Internationale de l'AIMS, Paris, 5-7 juin.

BOUSSARD V., (2008). Sociologie de la gestion : les faiseurs de performance, Perspectives sociologiques, Belin, Paris.

CAMPINOS-DUBERNET M. et JOUGLEUX M., (2002). La certification ISO 9000 dans le changement stratégique d'une bureaucratie de service, XI Conférence internationale de l'AIMS, 5-7 juin, EFCP-EAP, Paris.

CLAVERANNE J.P., VINOT D., FRAISSE S., ROBELET M., CANDEL D., DUBOIS D., MARCHAUDON P., (2003). Les perceptions de la qualité chez les professionnels des établissements de santé, Note de synthèse, DHOSS.

CONSTANDRIOPOULOS A. P., SOUTEYRAND Y., (éds) (1996). L'hôpital stratège. Dynamique locale et offre de soins, John Libbey Eurotext, Paris. 
CROZIER M. et FRIEDBERG E., (1977). Le phénomène bureaucratique, Le Seuil, Paris.

CUEILLE S. et RENUCCI A., (2000). Responsabilisation des acteurs dans les hôpitaux publics français et Management stratégique : analyse des dernières réformes juridiques, Politiques et Management Public 18 (2), 43-68.

DAVID A., (1998). Outils de gestion et dynamique du changement, Revue Française de Gestion 120, 44-59.

DECHAMP G. et ROMEYER C., (2005). L'appropriation du concept de gestion par des managers non gestionnaires, Colloque L'appropriation des outils de gestion : vers de nouvelles perspectives théoriques? Preactis, Université Jean Monnet, Saint-Étienne.

DE CERTEAU M., (1990). L'invention du quotidien, tome I. Arts de faire, Gallimard, Paris.

DE VAUJANY F.X. (ed.), (2005). De la conception à l'usage : l'appropriation des outils de gestion, Editions EMS, Cormelles-le-Royal.

GILBERT P., (1998). L'instrumentation de gestion : la technologie de gestion science humaine? Economica, Paris.

GRAWITZ M., (1996). Méthodes des sciences sociales 10e Ed., Dalloz, Paris.

GRIMAND A. (éd.), (2006). L'appropriation des outils de gestion : vers de nouvelles perspectives théoriques? Publications de l'Université de Saint-Étienne, Saint-Étienne.

HALGAND N., (2003). L'accréditation hospitalière : contrôle externe ou levier de changement?, Revue Française de Gestion 147, 219-231.

HATCHUEL A. et WEIL B., (1992). L'expert et le système, Economica, Paris.

HUSSER J., (2006). Le management stratégique de la qualité hospitalière par l'encadrement intermédiaire, $X V^{\mathrm{e}}$ Conférence Internationale de Management Stratégique, Annecy/Genève 13 au 13 juin.

LORINO P., (2002). Vers une théorie pragmatique et sémiotique des outils appliquée aux instruments de gestion, Essec Research Center, DR 02015.

LORINO P., TRICARD, B., \& CLOT, Y., (2011). Research Methods for Non-Representational Approaches to Organizational Complexity: The Dialogical Mediated Inquiry, Organization Studies, 32(6),769-801.

LOZEAU D., (2004). La démarche qualité : de l'enlisement d'une technique à l'enracinement d'un archétype, $X I I I^{\mathrm{e}}$ Conférence Internationale de l'AIMS, Normandie, Vallée de Seine 2, 3 et 4 juin.

MAUGERI S. (éd.), (2001). Délit de gestion, La Dispute, Paris.

MEYER J. W. \& ROWAN B., (1977). Institutionalized organizations: Formal structure as myth and ceremony, American Journal of Sociology 86, 340-363.

MILES M. B. \& HUBERMAN A.M., (1991). Analyse des données qualitatives, traduction édition américaine, De Boeck, Bruxelles.

MINVIELLE E., (2003). De l'usage des concepts gestionnaires dans le champ de la santé : le cas de la qualité hospitalière, Revue Française de gestion 146, 167-189.

MOISDON J.C. (ed.), (1997). Du mode d'existence des outils de gestion, Editions Seli-Arslan, Paris.

PASCAL C., (2003). La gestion par processus à l'hôpital entre procédure et création de valeur, Revue Française de Gestion 146, 191-204.

PIAGET J., (1967). La psychologie de l'intelligence, Coll. U2, Armand Colin, Paris.

RABARDEL P., (1997). Activités avec instruments et dynamique cognitive du sujet In MORO C., SCHNEUWLY B. et BROSSARD M. (ed.). Outils et signes : perspectives actuelles de la théorie de Vytgotski, Peter Lang, 35-49.

REYNAUD J.D., (1988). Les régulations dans les organisations : régulation de contrôle, Revue Française de Sociologie XXIX, 5-18.

VALETTE A., (1996). Une gestion stratégique à l'hôpital ?, Revue Française de Gestion 109, 92-100.

VERGNAUD G., (2001). Forme opératoire et prédicative de la connaissance, Actes du colloque GDM, Montréal, mai.

WEILL M., (2001). Le management de la Qualité, La Découverte, Paris.

YIN R. K., (2003). Case study research: Design and methods $3^{\text {th }}$ Ed., Sage Publishing, Beverly Hills. 


\section{Annexe \\ Architecture du manuel de certification HAS V2 - Edition 2007}

\begin{tabular}{|c|c|c|}
\hline Chapitre & Titre & Référence \\
\hline $\begin{array}{l}\text { Politique et qualité } \\
\text { du management }\end{array}$ & & $\begin{array}{l}\text { Les orientations stratégiques de l'établissement } \\
\text { La place du patient et de son entourage } \\
\text { La politique des ressources humaines } \\
\text { La politique du système d'information } \\
\text { et du dossier du patient } \\
\text { La politique de communication } \\
\text { La politique d'amélioration de la qualité } \\
\text { et de gestion des risques } \\
\text { La politique d'optimisation des ressources } \\
\text { et des moyens }\end{array}$ \\
\hline \multirow[t]{5}{*}{$\begin{array}{l}\text { Ressources } \\
\text { transversales }\end{array}$} & Ressources Humaines & La maîtrise des processus de GRH \\
\hline & $\begin{array}{l}\text { Fonctions hôtelières } \\
\text { et logistiques }\end{array}$ & $\begin{array}{l}\text { L'organisation et la maîtrise de la qualité } \\
\text { des fonctions hôtelières et logistiques }\end{array}$ \\
\hline & $\begin{array}{l}\text { Organisation de } \\
\text { la qualité et de la } \\
\text { gestion des risques }\end{array}$ & $\begin{array}{l}\text { Le management de la qualité } \\
\text { La gestion des risques } \\
\text { Le dispositif de veille sanitaire }\end{array}$ \\
\hline & $\begin{array}{l}\text { Qualité et sécurité de } \\
\text { l'environnement }\end{array}$ & $\begin{array}{l}\text { Le programme de surveillance et de prévention } \\
\text { du risque infectieux } \\
\text { La gestion des risques liés aux dispositifs } \\
\text { médicaux } \\
\text { La gestion des risques liés à l'environnement } \\
\text { La sécurité et la maintenance des infrastructures } \\
\text { et des équipements } \\
\text { La sécurité des biens et des personnes }\end{array}$ \\
\hline & $\begin{array}{l}\text { Système } \\
\text { d'information }\end{array}$ & Le système d'information \\
\hline $\begin{array}{l}\text { Prise en charge } \\
\text { du patient }\end{array}$ & Droits du patient & $\begin{array}{l}\text { L'information du patient } \\
\text { La recherche du consentement et des volontés } \\
\text { du patient } \\
\text { La dignité du patient et la confidentialité }\end{array}$ \\
\hline
\end{tabular}




\begin{tabular}{|c|c|c|}
\hline & Parcours du patient & $\begin{array}{l}\text { L'accueil du patient et de son entourage } \\
\text { La prise en charge du patient se présentant } \\
\text { pour une urgence } \\
\text { L'évaluation initiale de l'état de santé du patient } \\
\text { et le projet thérapeutique } \\
\text { personnalisé } \\
\text { Les situations nécessitant une prise en charge } \\
\text { adaptée } \\
\text { La prise en charge de la douleur } \\
\text { La continuité des soins } \\
\text { Le dossier du patient } \\
\text { Le fonctionnement des laboratoires } \\
\text { Le fonctionnement des secteurs d'imagerie } \\
\text { et d'exploration fonctionnelle } \\
\text { L'organisation du circuit du médicament } \\
\text { Le fonctionnement des secteurs d'activité } \\
\text { interventionnels } \\
\text { La radiothérapie } \\
\text { L'organisation du don d'organes ou de tissus } \\
\text { à visée thérapeutique } \\
\text { Les activités de rééducation et/ou de soutien } \\
\text { L'éducation thérapeutique du patient } \\
\text { La sortie du patient } \\
\text { La prise en charge du patient en soins palliatifs } \\
\text { Le décès du patient }\end{array}$ \\
\hline \multirow[t]{3}{*}{$\begin{array}{l}\text { Évaluations } \\
\text { et dynamiques } \\
\text { d'amélioration }\end{array}$} & $\begin{array}{l}\text { Pratiques } \\
\text { professionnelles }\end{array}$ & $\begin{array}{l}\text { L'évaluation de la pertinence des pratiques } \\
\text { des professionnels } \\
\text { L'évaluation des risques liés aux soins } \\
\text { L'évaluation de la prise en charge des pathologies } \\
\text { et des problèmes de santé principaux }\end{array}$ \\
\hline & $\begin{array}{l}\text { Les usagers et les } \\
\text { correspondants } \\
\text { externes }\end{array}$ & $\begin{array}{l}\text { L'évaluation de la satisfaction du patient, } \\
\text { de son entourage et des correspondants externes }\end{array}$ \\
\hline & $\begin{array}{l}\text { Politiques et } \\
\text { management }\end{array}$ & L'évaluation des politiques et du management \\
\hline
\end{tabular}

